\title{
Comparison of SDD/WDS/Micro-XRF Element Mapping Methods Using a Garnet Schist Example
}

\author{
Craig S. Schwandt
}

McCrone Associates, Inc., 850 Pasquinelli Drive, Westmont, IL 60559-5539

Advances in technology have introduced new data collection options like high count rate acquisition with silicon drift detectors, or the possibility of lower detection limits due to lower background generation with X-ray beam excitation. For some samples, such as compounds with a limited number of components, or materials with elements that do not have spectral overlaps, the new techniques offer great advantages in terms of acquisition speed and lower detection limits. However, for analysis of complex materials and especially analysis of trace elements, silicon drift detectors (SDD) and micro-XRF systems require careful attention during data collection otherwise the results may be compromised.

Previously, it has been shown that micro-XRF mapping can reveal elements at lower concentrations than is possible with lithium drifted silicon (SiLi) detector energy dispersive spectrometry (EDS) systems [1,2]. Micro-XRF mapping offers the option of quickly finding elements that normally occur in minor and trace level concentrations but which may occur at higher concentrations when they are essential structural constituent elements of minerals such as accessory mineral phases. In such instances it is not necessary to conduct a principal components phase analysis, as the key elements are at sufficient concentrations to make phases recognizable. For example, rare earth element phosphate minerals such as monazite and xenotime become quickly obvious (Fig. 1).

Similarly, SDD energy dispersive spectrometry systems permit the use of dramatically higher probe currents and acquire maps at phenomenal rates; this offers the possibility of detecting lower concentration elements especially with mapping [3]. For certain samples SDD technology proves extraordinary, because fast multispectral imaging permits multivariate statistical phase identification routines that can easily distinguish phases that might otherwise be overlooked [4].

The ultimate goal in elemental mapping is to have fully quantified maps that are fully background and ZAF matrix corrected using standard reference materials with compositions similar to the unknowns. However, regardless of the reduction models used, the critical issue is separating the peak of interest from the background. This is the critical aspect which must not be overlooked when using SDD and micro-XRF to analyze or map complex multi-component samples, because both methods can easily generate extensive sum and scattering peaks that affect the background. In such instances, the rapidly collected data can be extremely misleading or even incorrect [3,5]. Therefore, when mapping elements that occur at minor and trace element concentrations, the best results are still obtained by wavelength dispersive spectrometry (WDS). WDS may take considerably longer, but it offers accuracy in exchange.

As an example using a Black Hills, South Dakota, garnet schist (Fig. 1), the rare earth elements (REE) are of interest. The energy range of four to nine kilo-electron volts covering the L lines for the REE is therefore of interest. Figure 2 shows a maximum pixel spectrum for the data from Figure 1 collected using a micro-XRF/SiLi-EDS system. Compared to the sum spectrum for the entire map, interesting peaks are revealed; however, they are not at the proper energies to represent REE. Instead they likely represent multi-element sum peaks and Compton scattering peaks. Therefore, it 
is critical to not use high dead-time acquisition, or background effects may overwhelm the energy of interest, thus negating any fast acquisition benefit.

Interestingly, the same problem occurs with high probe current analysis with a SDD. If one is not careful, the formation of complex and multiple sum peaks easily boosts the background, overwhelming small peaks of interest. This is easily demonstrated by comparing Figures 3 and 4 . Figure 3 was collected at $10 \mathrm{nA}$ with the longest time-constant possible for the system $(12 \mathrm{~ms})$. Figure 4 was collected using $700 \mathrm{nA}$ and the shortest time-constant possible for the system $(0.5 \mathrm{~ms})$. The background of the spectrum is clearly distorted in the high current case (Fig. 4), and it is very similar to the micro-XRF/SiLi EDS spectrum of Figure 2.

Therefore, quick acquisition of spectra, whether electron or photo excited, must be accomplished with extreme care regarding the spectrum background relative to the data that is intended for extraction. Otherwise, the best results may still require using a WDS system, even though it takes longer.

\section{References}

[1] C.S. Schwandt, Microsc Microanal 15(Suppl 2) (2009) 34.

[2] Cross, B.J. et al. Proceedings of the International Microscopy Congress 16, (2006) Sapporo, Japan.

[3] D. Newbury and N. Ritchie, Microsc Microanal 15(Suppl 2) (2009) 6.

[4] P.G. Kotula and J.R. Michael, Microsc Microanal 12(Suppl 2) (2006) 1390.

[5] R. Wuhrer and K. Moran, Microsc Microanal 15(Suppl 2) (2009) 30.

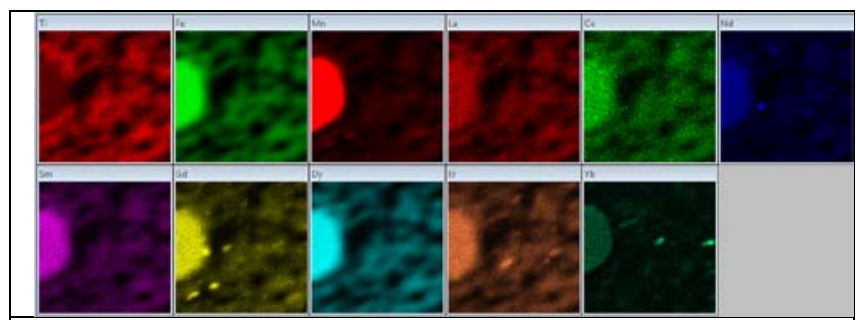

Figure 1: Micro-XRF acquired data showing major and REE maps for garnet schist.

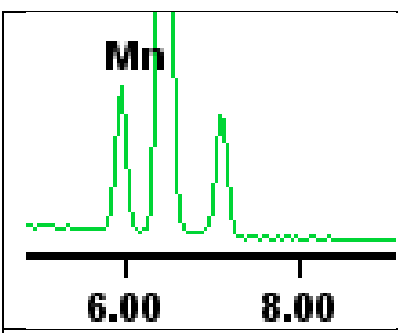

Figure 3: Portion of an SDD-EDS spectrum collected with low probe current and long time constant.

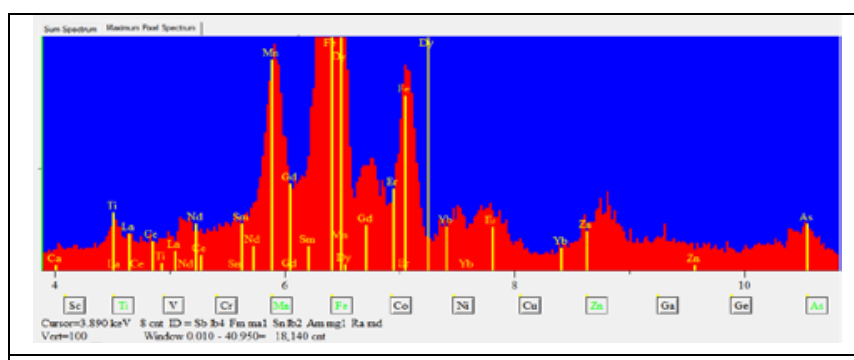

Figure 2: Maximum pixel spectrum from data shown in Figure 1.

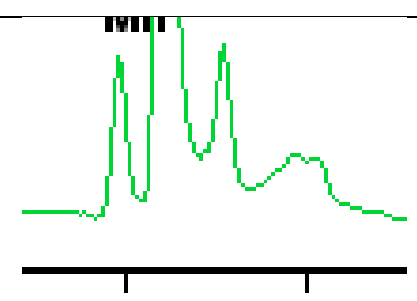

\section{$\mathbf{6 . 0 0} \quad 8.00$}

Figure 4: Portion of an SDD-EDS spectrum collected with high probe current and short time constant compared to Figure 3. 